\title{
The Case for Adding Randomness to the Nature-Nurture Debate
}

\author{
Stephen Rice, Associate Professor \\ Florida Institute of Technology \\ 150 W. University Blvd. Melbourne, FL 32901, USA \\ E-mail: scrice@ outlook.com \\ David Trafimow \\ New Mexico State University \\ Las Cruces, New Mexico, USA \\ Rian Mehta \\ Florida Institute of Technology \\ 150 W. University Blvd. Melbourne, FL 32901, USA
}

Received: October 26, 2015 Accepted: November 18, 2015 Published: November 20, 2015

doi:10.5296/iss.v3i2.8489 URL: http://d x.doi.org/10.5296/iss.v3i2.8489

\begin{abstract}
People dramatically underestimate the role that randomness plays in almost every aspect of human behavior. We show that the typical belief is that a combination of genetic inheritance and environment accounts for all of human behavior. However, in contrast to this belief, we review literature from the tradition of potential performance theory that shows that much human behavior, in a wide variety of domains, is due to randomness. The fact that randomness has been demonstrated to be an important contributor to human behavior, in many domains, suggests that psychologists should take it more seriously. Instead of attempting to account for human behavior solely with genetic and environmental influences, we argue that randomness should be included too. Thus, it is the interaction of genetics, environment, and randomness that produces human behavior, and behavioral researchers should recognize this and adjust their research programs accordingly.
\end{abstract}




\section{Macrothink

Keywords: nature, nurture, randomness, potential performance theory 2015, Vol. 3, No. 2 


\section{Introduction}

The question has often been asked, "which has more influence on human behavior - nature or nurture?" There are many proponents for each side. For example, studies involving identical twins (Bergeman, Chipuer, Plomin, \& Pedersen, 1993; Jang, Livesley, \& Vernon, 1996; Horowitz, Videon, Schmitz, \& Davis, 2003) and siblings (McGuire, Neiderhiser, Reiss, \& Hetherington, 1994; Plomin, Reiss, Hetherington, \& Howe, 1994; Roisman \& Fraley, 2008) show that nature (genetics) plays a huge role in how we develop. Twins who were separated at birth and met up later in life have a remarkable amount of similarities; these correlations are much higher than the general population (McCartney, Harris, \& Berbieri, 1990). Similar studies involving siblings also show higher similarities compared to the general population (Collins, Maccoby, Steainberg, Hetherington, \& Bornstein, 2000). Clearly, nature plays a strong role in how we develop and our future behavior.

On the other hand, nurture (environmental factors) has also been shown to play a strong role in human behavior. For example, the classic Stanford Prison Study (Zimbardo, 1971) showed that when participants were assigned to the roles of "guard" or "prisoner", they began to behave in the corresponding manner. "Guards" became more aggressive, while "prisoners" became more compliant. Interestingly, this change occurred so quickly that the study had to be terminated after only 6 days. Another example involves operant conditioning. Skinner (Skinner, 1963; Blum \& Kennedy, 1967; Shields \& Greder, 2003) showed that reward and punishment strongly influence behavior. Think of a slot machine and how effective it is in getting people to sit down and pull the handle over and over and over again, hoping that eventually they will get paid off. It is so effective that even the knowledge that one will lose money in the long run is not a deterrent to performing the behavior.

This nature-nurture argument occurs every semester in Psychology, Biology, Philosophy, etc., courses all around the world, without resolution. Sometimes nature plays a greater role whereas sometimes nurture plays a greater role. However, most people seem to agree that the combination of nature and nurture accounts for most, if not all, of human behavior. To test this concept, we asked 250 people from around the world via an online survey to tell us how much of each (nature and nature) accounted for human behavior. In about $90 \%$ of the cases, participants came to the conclusion that nature + nurture accounted for $100 \%$ of human behavior. But are they right?

What people apparently are not taking into account in their opinions is that there is a third factor. This factor is randomness. Now before we explain randomness, let us define consistency. In our terms, consistency is when a person performs in an identical manner when presented with multiple identical situations. For example, imagine a person takes a test with 50 multiple-choice questions. Then, the following day, they retake the exact same test with the exact same 50 questions. If they give the same answer to each question that they gave to that corresponding question in the previous test, then they are being consistent. If they give a different answer to the same question from the previous test, then they are being inconsistent. It is easy to determine how consistent they are overall by calculating a consistency coefficient, which is each person's correlation across the two test-taking occasions. This coefficient tells 
us how consistent the person is overall. The inverse of this value tells us how inconsistent, or random, they are.

Now the question we are interested in changes to, "which has more influence on human behavior-nature or nurture or randomness?" The goal of this paper is not necessarily to answer that question because it depends on the person and situation. Instead, the goal is to show that randomness plays a much stronger role in human behavior than previously thought. As you recall from the study we described above, about $90 \%$ of the group of people we sampled did not even consider randomness as a factor. We believe the results of previous experiments that have measured randomness will surprise them considerably.

Fortunately, a recent theoretical advance in Psychology-termed potential performance theory (PPT; Trafimow \& Rice, 2008)-has provided a mechanism for parsing apart systematic factors (e.g. skills, knowledge, strategies, etc.) from random factors (defined above). We will not go into great detail about how PPT accomplishes this (see Trafimow \& Rice, 2008; 2009 for the mathematical proofs). What we will do is go into detail regarding the empirical studies that these researchers and their colleagues have performed since the advent of PPT. These studies all share the fact that they involve human performance in one capacity or another. Furthermore, they were all designed in a way that allows for a PPT analysis on them; that is, the systematic and random factors have been parsed apart.

Each of the studies provides us with consistency coefficients, as described above. As a whole, we can make assumptions about randomness and its place in the nature-nurture-randomness debate. Overall, we will discuss about a dozen empirical papers, with a total of 27 consistency coefficient values that are meaningful to the current topic. The experiments range from simple cognitive tasks like enumeration, more complex visual search tasks, tasks where humans are aided by an automated device, to education-based tests.

There are at least two ways to think about the consistency coefficients to be presented. One way is to take them at face value. An alternative way is to convert them into coefficients of determination. A coefficient of determination, in this context, gives the amount of variance that can be accounted for in performance on one block of trials, by variance in performance on the other block of trials. The coefficient of determination can be computed simply by squaring the consistency coefficient. For example, if a person's consistency coefficient on a particular type of task is .8 , then that person's coefficient of determination is $(.8)(.8)=.64$. Thus, in this example, $64 \%$ of the person's variance in performance on one block of trials can be accounted for by his or her variance in performance on the other block of trials. If the coefficient of determination is then subtracted from unity, it gives a measure of the amount of variance that cannot be accounted for in performance on one block of trials from variance in performance on the other block of trials. To continue with the example, $1-.64=.36$, and so $36 \%$ of the person's variance in performance across trials in the type of task being studied is attributable to randomness. We will present consistency coefficients and percent randomness values. 


\section{Review of Empirical Studies}

The review focuses first on the many different cognitive tasks that have been studied. Hunt et al. (in press) presented participants with anywhere from four to nine dots on a computer screen in a speeded counting task. In Experiment 1, the dots were presented horizontally; in Experiment 2, they were presented randomly on the screen. In the easiest case, where only four digits were presented, mean consistency coefficients were relatively large $(M=.83$ in Experiment 1 and $M=.83$ in Experiment 2) and mean randomness percentages were relatively small $(M=31 \%$ and $M=31 \%)$, though even $31 \%$ randomness can be considered to be much more than the amount of randomness that most people assume. When we switch to the most difficult case, where nine digits were presented, mean consistency coefficients were relatively small $(M=.38$ in Experiment 1 and $M=.31$ in Experiment 2) and mean randomness percentages were relatively large $(M=86 \%$ and $M=90 \%)$. An interesting consequence of the difference in randomness across the different conditions is that it accounts, almost completely, for effects of number of digits presented on performance. There doesn't seem to be a lot of room for nature and nurture in the participants' performance when the task was difficult.

Rice et al. (2012) performed three experiments where task difficulty was manipulated in various ways. In Experiment 1, participants searched for an $\mathrm{O}$ among Qs, with set sizes of 20 (easy) and 40 (difficult). In Experiment 2, the set size was always 40; however, the contrast between the letters and the background was either high (easy) or low (difficult). In Experiment 3, participants searched for a helicopter in aerial images of Baghdad with either a low-clutter (easy) background or a high-clutter (difficult) background. When the task was easy, mean consistency coefficients were relatively large $(M=.61$ in Experiment $1, M=.40$ in Experiment 2, and $M=.67$ in Experiment 3) with mean randomness percentages being $63 \%, 84 \%$, and $55 \%$, respectively. Mean consistency coefficients were smaller in the difficult conditions ( $M=.36$ in Experiment $1, M=.30$ in Experiment 2, and $M=.46$ in Experiment 3), and there was more randomness $(M=87 \%, M=91 \%$, and $M=79 \%)$. Importantly, even when the task was easy, more than $50 \%$ of the variance in performance was explained by randomness. When the task was difficult, this percentage of variance accounted for jumped up dramatically.

Trafimow and Rice (2009) performed a similar aerial search task, where participants searched for enemy tanks in aerial photographs. The mean consistency coefficient was only .37 in this arguably difficult task, and so randomness was large (86\%).

Rice, Trafimow and Hunt (2010) performed a similar experiment, but this time participants were aided by an automated device, of varying reliabilities, indicating whether the weapon was present or absent. While the aid provided recommendations, participants were responsible for making the final decision. Rice et al. presented separate values indicating mean consistency coefficients when the automated device was false-alarm-prone and when it was miss-prone, and also when the task was easiest (highest reliability for the automated computer responses) and most difficult (lowest reliability for the automated computer responses). When the task was easiest, mean consistency coefficients tended to be relatively 
large when the automation was miss-prone $(M=.68)$ and false-alarm-prone $(M=.74)$. Percentages of randomness that correspond to these consistency coefficients are $M=46 \%$ and $M=55 \%$, respectively. When the task was most difficult, mean consistency coefficients were relatively low when the automation was miss-prone $(M=.28)$ or false-alarm-prone $(M$ $=.26)$, and percentages of randomness were relatively high $(M=92 \%$ and $M=93 \%$, respectively).

Rice et al. (2011) had participants perform a visual search task under different time constraints. Participants were allowed 1-6 seconds to perform the task, with increments of 1 second between each condition. In Experiment 1, they searched for the letter $\mathrm{O}$ among Qs and in Experiment 2 they searched for a helicopter in aerial images. In the easiest condition (6 second time constraint), consistency coefficients were fairly high in both experiments $(M$ $=.86$ in Experiment 1 and $M=.72$ in Experiment 2). Conversely, randomness was moderate ( $M=26 \%$ in Experiment 1 and $M=.48 \%$ in Experiment 2$)$. In the most difficult conditions (1 second time constraint), consistency coefficients were low in both experiments $(M=.39$ in Experiment 1 and $M=.35$ in Experiment 2). Conversely, randomness was considerable $(M=$ $85 \%$ in Experiment 1 and $M=.88$ in Experiment 2).

Rice and Trafimow (2012a) combined the use of an automated device with a time pressure manipulation to test the effects on visual search. They suggested that using a heuristic of compliance to the automation, under time pressure, might increase performance by decreasing randomness in responding. Participants searched for a helicopter in aerial photographs, while aided by a diagnostic device that provided recommendations as to whether the target was present or absent. Participants were responsible for the final decision. The authors reported mean consistency coefficients in both the time pressure $(M=.77)$ and no time pressure $(M=.61)$ conditions, and so mean randomness was $41 \%$ and $63 \%$, respectively.

In the area of Education, there were three articles that we were able to include in our review. Rice et al. (2011) tested 346 participants across a variety of educational and aptitude topics such as mathematics, geography, memory tests, and others. Participants were most consistent on the astronomy test $(M=.78)$ whereas they were least consistent on the spatial memory test $(M=.38)$. Mean randomness figures were $39 \%$ for astronomy and $86 \%$ for spatial memory.

Rice, Trafimow and Kraemer (2012) had participants take a world history test spanning thousands of years and various countries. The authors reported a mean consistency coefficient of .69 for the test, with the mean randomness being 52\%. After the participants were allowed to practice taking the test multiple times, the mean consistency coefficient increased somewhat to. 85 for the test, with the mean randomness being $28 \%$.

Rice and Trafimow (2012b) had Indian and American participants take an algebra test in order to compare consistency levels across the two countries. Indians were much more consistent than were Americans $(M=.80$ and $M=.64)$, which means that Indians were less random than were Americans $(M=36 \%$ and $M=59 \%)$. Rice and Trafimow showed that the difference in randomness accounts for the better performance of Indians over Americans in that particular test. 
Because PPT originally was developed in the context of moral decision-making, it would make sense that there would be an extensive literature on using PPT to investigate moral decision-making. Nevertheless, we are aware of only two such studies. Trafimow and Rice (2008) presented participants with scenarios where they had to decide whether a particular behavior was moral or immoral. Their participants were surprisingly consistent $(M=.80)$, and so randomness was relatively low $(M=36 \%)$, though still much higher than common sense seems to suggest.

Trafimow et al. (2011) performed an additional study that was not specifically about participants' own moral decisions, but rather about their expectations about the moral decisions others would make. There were two types of moral items and these were based on Kant's (1797/1991) distinction between perfect and imperfect duties. According to Kant, moral people always have to perform perfect duties whereas moral people do not always have to perform imperfect duties (see Korsgaard, 1985; Trafimow \& Trafimow, 1999; Trafimow et al, 2005 for relevant descriptions and research). Interestingly, consistency coefficients were not very different for perfect and imperfect duties $(M=.55$ and $M=.57)$, and randomness was large in both cases $(M=70 \%$ and $M=68 \%)$.

\section{Discussion}

The foregoing review suggests that randomness is an issue that should be taken seriously. Nature and nurture (systematic factors) simply do not account for all of human behaviors, even in the "easy" tasks. We wish to note that the review does not specify exactly how much of human behavior is caused by randomness, because while we had 1196 participants across a myriad of tasks, it is impossible to generalize this data to the entire human population. More research needs to focus on replicating these findings in other tasks.

Additionally, these data do not necessarily suggest that randomness always occurs independently of nature and nurture. It is commonly believed among scientists that nature and nurture interact with each other (Lerner, 1978; Rutter, 1997; McClearn, 2003). For example, one might have a latent gene that is triggered by an environmental event. If the environmental event never occurs, the gene may lay dormant the person's entire life (Lichtenstein \& Pedersen, 1995; Lewis \& Levitt, 2002; Lahiri, Maloney, Riyaz, Wen Ge, \& Zawia, 2007). This same phenomenon is possible for randomness. Nature and nurture may independently, or inter-dependently, interact with randomness. For example, it may be the case that people are predisposed genetically to randomness (Trafimow \& Rice, 2015).

Or it may be that environmental factors cause people to become more or less random throughout their lives. Or perhaps there could be a case of environmental factors triggering genetic changes that in turn cause more or less randomness. If we find that these factors do influence the amount of randomness, then it seems plausible that we can decrease or eliminate randomness by reversing the process. Further research should be conducted to determine the interactive role of randomness with nature and nurture.

There is substantial variation in randomness across all the tasks, and the conditions under which those tasks were performed. This leads us to believe that randomness fluctuates as a 
function of what type of task a person is doing. Furthermore, although we were not able to convey this before in a convenient manner, we wish to point out that there also is substantial variation in randomness associated with particular individuals. For the sake of brevity, we presented mean consistency coefficients but these means obscure the fact that in many of the articles we reviewed, there were specific people who had consistency coefficients ranging from being indistinguishable from zero (complete randomness) to being close to or equal to one (no randomness). So randomness can be influenced not only by the type of task, and the conditions under which the task is performed, but also by who is doing the task.

Given that there is substantial randomness in human behavior, why should we care? One reason for caring is that the fact is interesting in its own right, particularly as it contradicts the common intuition that all human behavior is determined by systematic factors. A second reason harks back to the issue of nature versus nurture. The evidence from the review suggests that the issue needs to be reframed: It is not about nature plus nurture, but rather about nature plus nurture plus randomness. This reframing brings up a host of issues for researchers to consider. Is the randomness we have uncovered "fundamental" in that it reflects how the human universe happens to be or does it reflect that there is much that merely "looks" random because of insufficient knowledge but is not fundamental? Is the degree of human randomness itself a function of nature and nurture; if nature and nurture are sufficiently favorable, does that decrease randomness in human behavior? How about comparisons to nonhumans; is human behavior more random or less random than the behavior of other animals? Does human versus nonhuman randomness itself depend on the type of task being performed? We could go on but it should be obvious that there is plenty of grist for both psychological and philosophical research mills to run for a long time.

Another reason to care about randomness in human behavior is that, as Trafimow and Rice (2008) pointed out, it has important implications for the ability of humans to successfully perform tasks. Because people seem to find this to not be intuitive, some explanation is in order. Imagine the ideal condition where a person has perfect knowledge and ability to perform a task perfectly, and also that her behavior is not subject to randomness. This person's performance should be at the $100 \%$ success level. But now, let's add some randomness by supposing that on 2 out of every hundred trials, the person's response is decided by a coin flip. In that case, our statistical expectation is that the person would perform at a $99 \%$ success level rather than at the $100 \%$ success level she would achieve when there is no coin flipping involved. Clearly, as there are coin flips on more trials, randomness will increase, and task performance will decrease. In the extreme case, where there is a coin flip on each trial, the expectation is that the person should perform at the chance level. For a dichotomous task of the types used in the literature we reviewed, the expectation would be that the person would perform at the $50 \%$ success level. In general, then, the ultimate applied reason for caring about randomness is that it sets a limit on human performance; more randomness implies that performance is reduced to become closer to chance. Furthermore, if employers wish to gain realistic estimates about how their employees will perform, randomness assessments will have to be an important part of the assessment process. 


\section{References}

Bergeman, C. S., Chipuer, H. M., Plomin, R., \& Pedersen, N. L. (1993). Genetic and environmental effects on openness to experience, agreeableness, and conscientiousness: An adoption/twin study. Journal of Personality, 61(2), 159-179. http://dx.doi.org/10.1111/j.1467 -6494.1993.tb01030.x

Blum, E. R., \& Kennedy, W. A. (1967). Modification of Dominant Behavior in School Children. Journal of Personality And Social Psychology, 7(3), 275-281. http://dx.doi.org/10.1037/h0025071

Collins, A. W., Maccoby, E. E., Steainberg, L., Hetherington, M. E., \& Bornstein, M. H. (2000). Contemporary research on parenting: The case for nature and nurture. American Psychologist, 55(2), 218-232. http//dx.doi.org/10.1037/0003-066X.55.2.218

Horowitz, A. V., Videon, T. M., Schmitz, M. F., \& Davis, D. (2003). Rethinking twins and environments: Possible social sources for assumed genetic influences in twin research. Journal of Health and Social Behavior, 44(2), 111-129. http://dx.doi.org/10.2307/1519802

Hunt, G., Rice, S. Trafimow, D., \& Sandry, J. (in press). Using Potential Performance Theory to analyze systematic and random factors in enumeration tasks. American Journal of Psychology. http//dx.doi.org/10.5406/amerjpsyc.126.1.0023

Jang, K. L., Livesley, W., \& Vernon, P. A. (1996). Heritability of the big five personalitydimensions and their facets: A twin study. Journal of Personality, 64(3), 577-591. http://dx.doi.org/10.1111/j.1467-6494.1996.tb00522.x

Kant, I. (1991). The metaphysics of morals (M. Gregor, Trans.). Cambridge, UK: Cambridge University Press. (Original work published 1797).

Korsgaard, C. M. (1985). Kant's formula of universal law. Pacific Philosophical Quarterly, 66, 24-47.

Lahiri, D. K., Maloney, B., Riyaz B., Wen, G. Y., \& Zawia, N. H. (2007). How and When Environmental Agents and Dietary Factors Affect the Course of Alzheimer's Disease: The "LEARn" Model (Latent Early-Life Associated Regulation) May Explain the Triggering of AD. Current Alzheiner Research, 4(2), 219-228. http://dx.doi.org/10.2174 /156720507780362164

Lerner, R. M. (1978). Nature, Nurture, and dynamic interactionism. Human Development, 21(1), 1-20. http://dx.doi.org/10.1159/000271572

Lewis, D. A., \& Levitt, P. (2002). Schizophrenia as a disorder of Neurodevelopment. Annual Review of Neuroscience, 25, 409-432. http://dx.doi.org/10.1146 /annurev.neuro.25.112701.142754

Lichtenstein, P., \& Pedersen, N. L. (1995). Social relationships, stressful life events, and self-reported physical health: Genetic and environmental influences. Psychology \& Health, 10(4), 295-319. http://dx.doi.org/10.1080/08870449508400245 


\section{Macrothink}

Issues in Social Science ISSN 2329-521X 2015, Vol. 3, No. 2

McCartney, K., Harris, M. J., \& Berbieri, F. (1990). Growing up and growing apart. Psychological Bulletin, 107, 226-237. http://dx.doi.org/10.1037/0033-2909.107.2.226

McClearn, G. E. (2003, June 19). Nature and nurture: Interaction and coactions American Journal of Medical Genetics Part B: Neuropsychiatric Genetics, 124B(1), 124-130. http://dx.doi.org/10.1002/ajmg.b.20044

McGuire, S., Neiderhiser, J. M., Reiss, D., \& Hetherington, E. (1994). Genetic and environmental influences on perceptions of self-worth and comptence in adolescence: A study of twins, full siblings, and step-siblings. Child Development, 65(3), 785-799. http:/dx.doi.org/10.2307/1131418

Plomin, R., Reiss, D., Hetherington, E., \& Howe, G. W. (1994). Nature and nurture: Genetic contributions to measures of the family environment. Developmental Psychology, 30(1), 32-43. http://dx.doi.org/10.1037/0012-1649.30.1.32

Rice, S., \& Trafimow, D. (2012b). Using Potential Performance Theory to assess differences in math abilities between citizens from India and the United States. Higher Education Studies, 2(3), 24-29. http://dx.doi.org/10.5539/hes.v2n3p24

Rice, S., \& Trafimow, D. (2012a). Time pressure heuristics can improve performance due to increased consistency: A PPT methodology. Journal of General Psychology, 139(4), 273-288. http://dx.doi.org/10.1080/00221309.2012.705187

Rice, S., Geels, K., Hackett, H., Trafimow, D., McCarley, J. S., Schwark, J., \& Hunt, G. (2012). The harder the task, the more inconsistent the performance: A PPT analysis on task difficulty. Journal of General Psychology, 139(1), 1-18. http://dx.doi.org/10.1080/00221309.2011.619223

Rice, S., Geels, K., Trafimow, D., \& Hackett, H. (2011). Our students suffer from both lack of knowledge and consistency: A PPT analysis of test-taking. US-China Education Review, 1(6), 845-855.

Rice, S., Trafimow, D., \& Hunt, G. (2010). Using PPT to analyze sub-optimal human-automation performance. Journal of General Psychology, 137(3), 310-329. http://dx.doi.org/10.1080/00221301003645236

Rice, S., Trafimow, D., \& Kraemer, K. (2012). Using Potential Performance Theory to assess how to increase student consistency in taking exams. Higher Education Studies, 2(4), 68-74. http:/dx.doi.org/10.5539/hes.v2n4p68

Rice, S., Trafimow, D., Keller, D., Hunt, G., \& Geels, K. (2011). Using PPT to correct for inconsistency in a speeded task. The Journal of General Psychology, 138(1), 12-34. http://dx.doi.org/10.1080/00221309.2010.531791

Roisman, G. I., \& Fraley, R. (2008). A behavior-genetic study of parenting quality, infant attachment security, and their covariation in a nationally representative sample. Developmental Psychology, 44(3), 831-839. http://dx.doi.org/10.1037/0012-1649.44.3.831 


\section{Macrothink}

Issues in Social Science ISSN 2329-521X 2015, Vol. 3, No. 2

Rutter, M. L. (1997). Nature-nurture integration: The example of antisocial behavior. American Psychologist, 52(4), 390-398. http·/dx.doi.org/10.1037/0003-066X.52.4.390

Shields, C., \& Gredler, M. (2003). A problem-solving approach to teaching operant conditioning. Teaching Of Psychology, 30(2), 114-116. http://dx.doi.org/10.1207 /S15328023TOP3002_06

Skinner, B. F. (1963). Operant behavior. American Psychologist, 18(8), 503-515. http://dx.doi.org/10.1037/h0045185

Trafimow, D., \& Rice, S. (2008). Potential performance theory (PPT): A general theory of task performance applied to morality. Psychological Review, 115(2), 447-462. http://dx.doi.org/10.1037/0033-295X.115.2.447

Trafimow, D., \& Rice, S. (2009). Potential Performance Theory (PPT): Describing a methodology for analyzing task performance. Behavior Research Methods, 41(2), 359-371. http://dx.doi.org/10.3758/BRM.41.2.359

Trafimow, D., \& Rice, S. (2011). Using a sharp instrument to parse apart strategy and consistency: An evaluation of PPT and its assumptions. Journal of General Psychology, 138(3), 169-184. http://dx.doi.org/10.1080/00221309.2011.574173

Trafimow, D., \& Rice, S. (2015). Is consistency a domain-general individual differences characteristic? Journal of General Psychology, 142(1), 1-22. http://dx.doi.org /10.1080/00221309.2014.961999

Trafimow, D., \& Trafimow, S. (1999). Mapping perfect and imperfect duties onto hierarchically and partially restrictive trait dimensions. Personality and Social Psychology Bulletin, 25, 687-697. http://dx.doi.org/10.1177/0146167299025006004

Trafimow, D., Bromgard, I. K., Finlay, K. A., \& Ketelaar, T. (2005). The role of affect in determining the attributional weight of immoral behaviors. Personality and Social Psychology Bulletin, 31, 935-948. http//dx.doi.org/10.1177/0146167204272179

Trafimow, D., Hunt, G. Rice, S., \& Geels, K. (2011). Using potential performance theory to test five hypotheses about meta-attribution. Journal of General Psychology, 138(2), 1-13. http://dx.doi.org/10.1080/00221309.2010.540591

Trafimow, D., MacDonald, J. A., \& Rice, S. (2012). Using PPT to account for randomness in perception. Attention, Perception, \& Psychophysics, 74, 1355-1365. http://dx.doi.org /10.3758/s13414-012-0319-7

Zimbardo, P. G. (1971). On the ethics of intervention in human psychological research: With special reference to the Stanford prison experiment. Cognition, 2(2), 243-256. http://dx.doi.org/10.1016/0010-0277(72)90014-5 